\title{
Color representation and interpretation of special effect coatings
}

\author{
A. Ferrero, ${ }^{1, *}$ E. Perales, ${ }^{2}$ A. M. Rabal, ${ }^{1}$ J. Campos, ${ }^{1}$ F. M. Martínez-Verdú, ${ }^{2}$ E. Chorro, ${ }^{2}$ and A. Pons ${ }^{1}$ \\ ${ }^{1}$ Instituto de Óptica, Consejo Superior de Investigaciones Científicas, c/Serrano 144, Madrid 28006, Spain \\ ${ }^{2}$ Department of Optics, Pharmacology and Anatomy, University of Alicante, \\ Carretera de San Vicente del Raspeig s/n 03690, Alicante, Spain \\ ${ }^{*}$ Corresponding author: alejandro.ferrero@csic.es
}

Received July 31, 2013; revised November 8, 2013; accepted December 13, 2013;

posted December 19, 2013 (Doc. ID 194877); published January 31, 2014

A representation of the color gamut of special effect coatings is proposed and shown for six different samples, whose colors were calculated from spectral bidirectional reflectance distribution function (BRDF) measurements at different geometries. The most important characteristic of the proposed representation is that it allows a straightforward understanding of the color shift to be done both in terms of conventional irradiation and viewing angles and in terms of flake-based parameters. A different line was proposed to assess the color shift of special effect coatings on $\mathrm{a}^{*}, \mathrm{~b}^{*}$-diagrams: the absorption line. Similar to interference and aspecular lines (constant aspecular and irradiation angles, respectively), an absorption line is the locus of calculated color coordinates from measurement geometries with a fixed bistatic angle. The advantages of using the absorption lines to characterize the contributions to the spectral BRDF of the scattering at the absorption pigments and the reflection at interference pigments for different geometries are shown. (C) 2014 Optical Society of America

OCIS codes: $\quad$ (330.1710) Color, measurement; (330.1720) Color vision; (290.1483) BSDF, BRDF, and BTDF. http://dx.doi.org/10.1364/JOSAA.31.000436

\section{INTRODUCTION}

The color of special effect coatings [1, 2$]$ ] has a strong dependence on the irradiation and viewing angles, which makes them present a very appealing appearance. As a consequence, they have become very popular in the automotive industry [3] and in other markets, such as cosmetics or in applications as security inks. Special effect coatings consist of a transparent substrate having embedded traditional color pigments, which absorb part of the light, and flake-shaped interference pigments. They have a selective reflection due to their layered structure which gives rise to interferences, the main origin of the special effect in the coatings. This is the most important difference with respect to the effect coatings, which have metallic flakes that specularly reflect the light. The perceived difference between effect coatings and special effect coatings is the much stronger variation of hue of the latter with respect to the former.

The increasing popularity of special effect coatings demands the development of new techniques and instruments to characterize their color [4-15]. Of particular relevance is to determine a reduced set of measurement geometries to completely characterize these coatings.

Concepts introduced by Cramer and co-workers [16-21] have contributed to the understanding of the color shift of effect and special effect coatings on $a^{*}, b^{*}$-diagrams. An interference line is defined as the locus of calculated color coordinates from measurement geometries with a fixed aspecular angle (the angular distance with respect to the specular direction), and an aspecular line as the locus of calculated color coordinates from measurement geometries with a fixed irradiation angle. According to the experience of the authors, interference lines with low aspecular angles characterize the special effect pigments (the strong color shift), whereas aspecular lines may characterize the scattering by absorption pigments.

Kirchner and Cramer tested these and other conclusions in a key paper on the subject [22]. They propose an interpretation of special effect coatings based on the incidence angle on the flakes and on the orientation of the viewed flakes (flake-based parameters) instead of using the conventional instrumental irradiation and viewing angles with respect to the coating surface. These flaked-based parameters better explain the color variation due to the interference pigments, since this variation depends on the incidence angle with respect to the flake $\left(\theta_{\text {inc }}\right)$ more than with respect to the coating $\left(\theta_{i}\right)$. The other flake-based parameter to be considered is the orientation angle of the flake with respect to the surface of the coating $\theta_{\text {flake }}$. As shown in Fig. 1, within the incident plane, a given $\left[\theta_{\text {inc }}, \theta_{\text {flake }}\right]$ pair defines the directions of irradiation $\left(\theta_{i}\right)$ and viewing $\left(\theta_{s}\right)$, which can be reciprocally interchanged.

The authors conclude that the claim with respect to the interference lines to characterize interference pigments is confirmed but not the claim with respect to the capacity of the aspecular lines to characterize absorption pigments. The reason why it happens is that the aspecular angle $\theta_{\text {asp }}$ is almost directly related to the physical flaked-based parameter $\theta_{\text {flake }}$, but $\theta_{i}$ does not have such a link to the flaked-based parameter $\theta_{\text {inc. }}$. Therefore, interference lines almost resemble the locus of calculated color coordinates from measurement geometries with a fixed $\theta_{\text {flake }}$ angle at different incidence angles on the flakes (assessment of the color shift at constant flake-produced chroma), whereas aspecular lines represent a color shift without a clear relation to the incidence angle on the flake and, in consequence, they do not allow the impact of the absorption pigments from the impact of the interference 


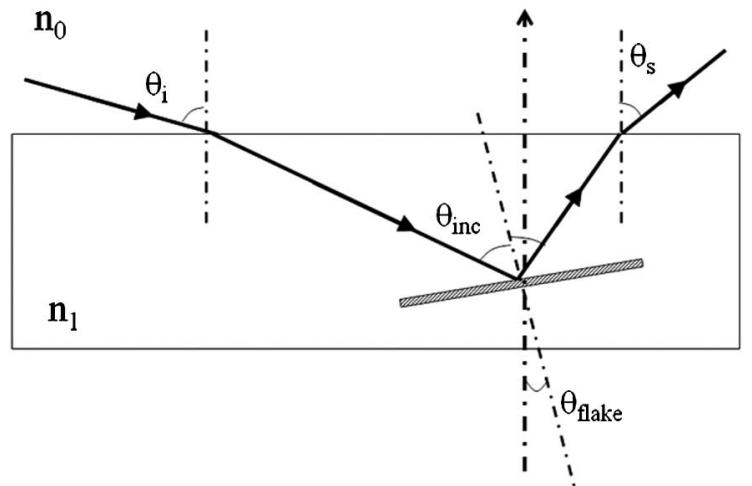

Fig. 1. Geometrical variables needed to describe the flake-related reflection mechanism.

flakes to be distinguished. Kirchner and Cramer [22] think, and we agree with them, that another set of measurement geometries should make a better characterization of the absorption pigments than the aspecular lines.

We have recently used a physical model based on the $\left[\theta_{\text {inc }}, \theta_{\text {flake }}\right]$ parameters to obtain a reduced and nonredundant set of measurement geometries to completely characterize, by a multiangle spectrophotometer, the spectral BRDF of any gonioapparent sample [23], which means that the spectral reflectance at any irradiation/viewing geometry can be estimated from the spectral BRDF measured at the proposed measurement geometries. This estimation is accomplished by using a principal components analysis (PCA) procedure $[24,25]$ to determine the spectral reflectance of a representative flake and the orientation distribution of the flakes $[D$ $\left.\left(\theta_{\text {flake }}\right)\right]$ [23]. It corroborated that the flake-based perspective is highly important to understand the color shift of special effect coatings.

The motivation of this work was to find a clear and simple flake-based representation of the color gamut of these coatings aligned with the previous concepts developed so far. For practical reasons, we consider it very important that the flake-based representation was easily connected to the conventional instrumental measurement geometries. This representation of the color gamut is presented in detail and it is demonstrated for real special effect coatings.

\section{PROPOSED REPRESENTATION}

The proposed representation of the color of special effect coatings is only based on in-plane geometries, since it has been observed that measurements at out-of-plane geometries do not contribute additional information to the characterization of the color [23]. Both irradiation angles $\left(\theta_{i}\right)$ and viewing angles $\left(\theta_{s}\right)$ are defined with respect to coating normal, where the projection of the irradiation direction on the sample is the origin of the azimuthal angles $\left(\phi_{i}\right.$ and $\left.\phi_{s}\right)$. Irradiation angles are positive by definition, whereas viewing angles are positive if they are on the same side of the sample surface normal as the irradiation direction $\left(\phi_{s}-\phi_{i}=0^{\circ}\right)$ and negative otherwise $\left(\phi_{s}-\phi_{i}=180^{\circ}\right)$, as prescribed in ASTM standards [26].

The representation is a color gamut table where the measurement geometry is given by the rows $\left(\theta_{i}\right)$ and the columns $\left(\theta_{s}\right)$ (see Fig. 2). Therefore, rows correspond to aspecular lines and diagonals from the top left to the bottom right correspond to interference lines, where the aspecular angle $\theta_{\text {asp }}$

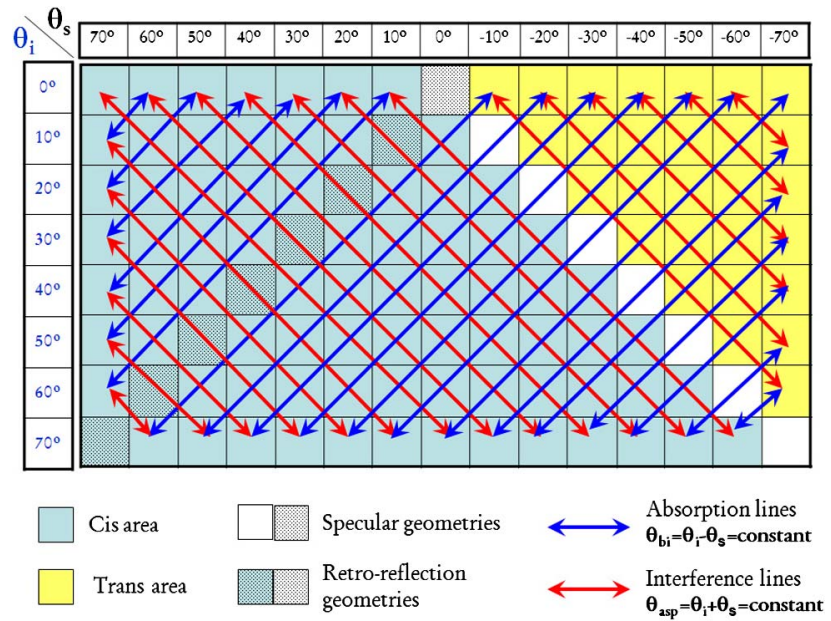

Fig. 2. Proposed representation of the color gamut for special effect coatings.

is calculated as $\theta_{i}+\theta_{s}$, being $\theta_{i}=-\theta_{s}$ the condition for the specular geometry. Cis $\left(\theta_{\text {asp }}>0^{\circ}\right)$ and trans geometries $\left(\theta_{\text {asp }}<0^{\circ}\right)$ are represented in different colored areas in Fig. 2. Every cell of the table contains a central square with the reproduction of the color in the corresponding geometry. The background of the cell contains the reproduction of the color but keeps the lightness for every geometry constant in order to clearly show the attainable hues [see Figs. 4(a)-9(a)].

Along the length of one interference line, the maximum hue variation due to special effect pigments is found, and this hue variation in the coating is better perceived the closer the geometry is to a specular geometry, that is, at small aspecular angles. The reason is that the orientation distribution of the flakes is peaked at $\theta_{\text {flake }}=0^{\circ}$, that causes more flakes to contribute to the reflected light near the specular angle. On the other hand, the display of the color of some coatings using the proposed representation [Figs. 4(a)-9(a)] made us think that the minimum hue variation due to interference pigments should be produced at perpendicular lines to the interference lines, that is, at the diagonals from the bottom left to the top right. These lines are defined as a constant difference $\theta_{i}-\theta_{s}$. This difference is named, using terms from radar science or remote sensing, the bistatic angle $\left(\theta_{\mathrm{bi}}\right)$, which is the angle between emission, target, and receptor. The retro-reflection condition is given at $\theta_{i}=\theta_{s}\left(\right.$ or $\theta_{\mathrm{bi}}=0^{\circ}$ ), but more importantly, it corresponds to the constant value $\theta_{\text {inc }}=0^{\circ}$, that would define a single spectral reflectance of the interference flakes (constant hue). In the same way, another single spectral reflectance may be defined for another given bistatic angle $\theta_{\mathrm{bi}}>0^{\circ}$, as long as the values of this angle were related to $\theta_{\mathrm{inc}}$.

In order to confirm this hypothesis, the almost linear relation between $\theta_{\mathrm{bi}}$ and $\theta_{\mathrm{inc}}$ is shown in Fig. 3(a), where a refractive index $\left(n_{\text {coat }}\right)$ of constant 1.5 was assumed for the coating, which is correct for most coatings. The calculation of the flake-based parameters from $\theta_{i}$ and $\theta_{s}$ was accomplished by using Eqs. (1) and (2) in the reference of Kirchner and Cramer [22] but dispensing with the signs

$$
\theta_{\text {flake }}=\frac{1}{2}\left|\arcsin \left(\frac{n_{\text {air }}}{n_{\text {coat }}} \sin \theta_{s}\right)+\arcsin \left(\frac{n_{\text {air }}}{n_{\text {coat }}} \sin \theta_{i}\right)\right|,
$$




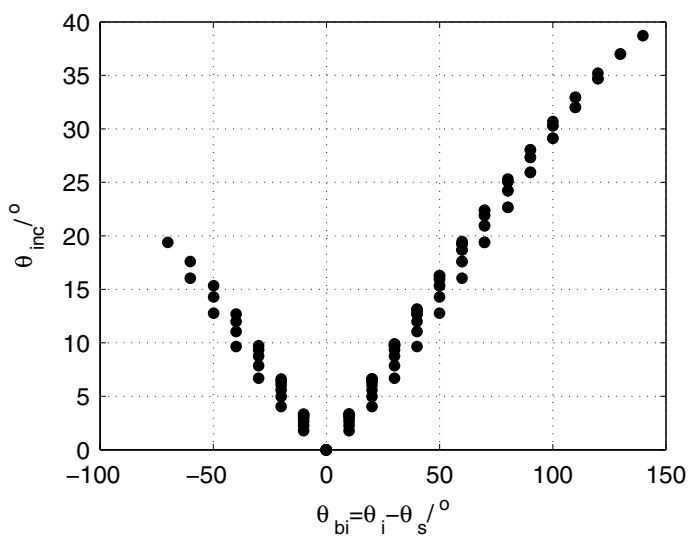

(a)

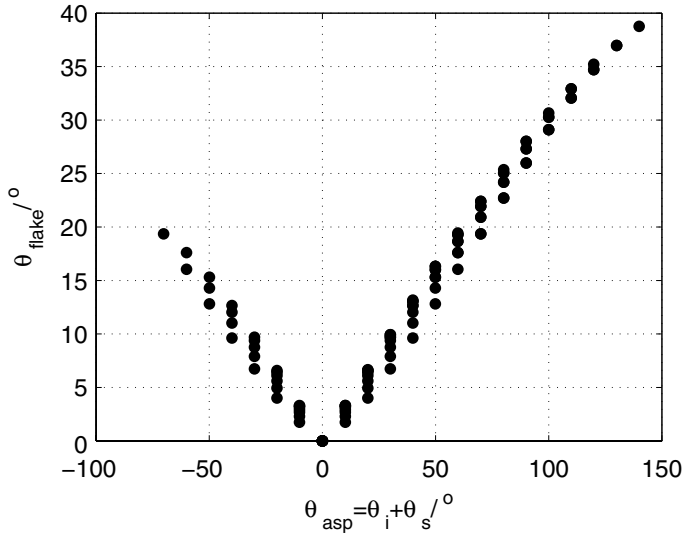

(b)

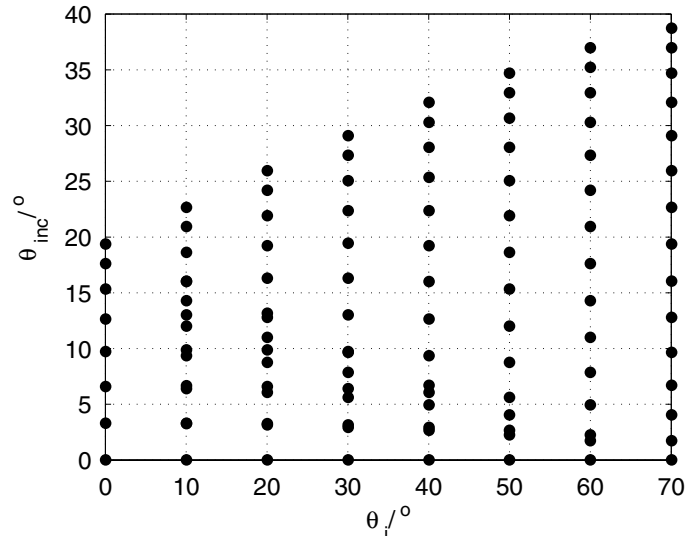

(c)

Fig. 3. Relation between flake-based parameters $\theta_{\text {inc }}$ and $\theta_{\text {flake }}$ and conventional measurement angles $\theta_{i}$ and $\theta_{s}$. (a) $\theta_{\text {inc }}$ versus $\theta_{\text {bi }}$, (b) $\theta_{\text {flake }}$ versus $\theta_{\text {asp }}$, and (c) $\theta_{\text {inc }}$ versus $\theta_{i}$.

$$
\theta_{\text {inc }}=\frac{1}{2}\left|-\arcsin \left(\frac{n_{\text {air }}}{n_{\text {coat }}} \sin \theta_{s}\right)+\arcsin \left(\frac{n_{\text {air }}}{n_{\text {coat }}} \sin \theta_{i}\right)\right| .
$$

Since we used absolute values of $\theta_{\text {inc }}$, there is a change of the slope sign at retro-reflection conditions. Given a value for $\theta_{\mathrm{bi}}$, the value of $\theta_{\text {inc }}$ can be predicted within less than a $4^{\circ}$ range. This is the tolerance of the approach of considering the lines of constant $\theta_{\mathrm{bi}}$ as the lines with less hue variation due to interference pigments. The relation between $\theta_{\text {asp }}$ and $\theta_{\text {flake }}$ [Fig. $\underline{3(\mathrm{~b})}$ ] is exactly the same as the previous one and the same considerations can be applied. The conclusion is that the interpretation of the color in terms of bistatic angles and aspecular angles is equivalent to use flake-based parameters. It is intuitive because it is based on the optics, unlike the instrument-based interpretation in terms of $\theta_{i}$ and $\theta_{s}$. The complete decorrelation between $\theta_{i}$ and $\theta_{\text {inc }}$
[Fig. 3(c)] implies that conclusions from aspecular lines are not immediate.

According to this reasoning, we suggest using the lines of constant $\theta_{\mathrm{bi}}$ to assess the maximum variation of special effect chroma (at constant flake-produced hue) in the coating. Similar to the interference lines where all possible interferences produced in the flakes are assessed at almost constant flake-produced chroma, we name it hereafter absorption lines, since, as we will show, they assess the different proportion of light absorbed at the absorption pigments with respect to the light reflected at interference pigments at constant incidence angle $\theta_{\text {inc }}$.

We propose an interpretation based on $\theta_{\mathrm{bi}}$ and $\theta_{\text {asp }}$ because they easily relate flake-based to instrument-based angles through the simple arithmetic

$$
\theta_{\text {flake }} \sim \theta_{\text {asp }},
$$

Table 1. Description of Interference, Absorption, and Aspecular Lines for the Interpretation of the Color Shift of Special Effect Coatings

\begin{tabular}{lll}
\hline Lines & \multicolumn{1}{c}{ Locus } & \multicolumn{1}{c}{ Description } \\
\hline Interference & $\theta_{\text {asp }}=\theta_{i}+\theta_{s}=$ constant $^{a}$ & Hue angle variation with almost fixed $\theta_{\text {flake }}$ (contribution from the same number of flakes). \\
Absorption & $\theta_{\mathrm{bi}}=\theta_{i}-\theta_{s}=$ constant $^{a}$ & Chroma variation with almost fixed $\theta_{\text {inc }}$ (constant spectral reflectance of the flakes). \\
Aspecular & $\theta_{i}=$ constant & Something between interference and absorption lines. \\
\hline
\end{tabular}


Table 2. Special Effect Coatings Studied in this Work

\begin{tabular}{llll}
\hline Code & Company & Description & Type of Pigment \\
\hline MCS1 & Merck & T20-04 WNT lapis sunlight & Colorstream \\
MCS2 & Merck & T20-02 WNT arctic fire & Colorstream \\
MXi & Merck & Light yellow and solaris red & ${\text { Xirallic } \mathrm{Al}_{2} \mathrm{O}_{3} / \mathrm{TiO}_{2}}$ \\
BASF1 & BASF coatings & Burgundy and olive green & Colorstream $^{a}$ \\
BASF2 & BASF coatings & Laurel green and greenish blue & Colorstream \\
BASF3 & BASF coatings & Light blue and pale turquoise & \\
\hline
\end{tabular}

${ }^{a}$ Visual description by authors.

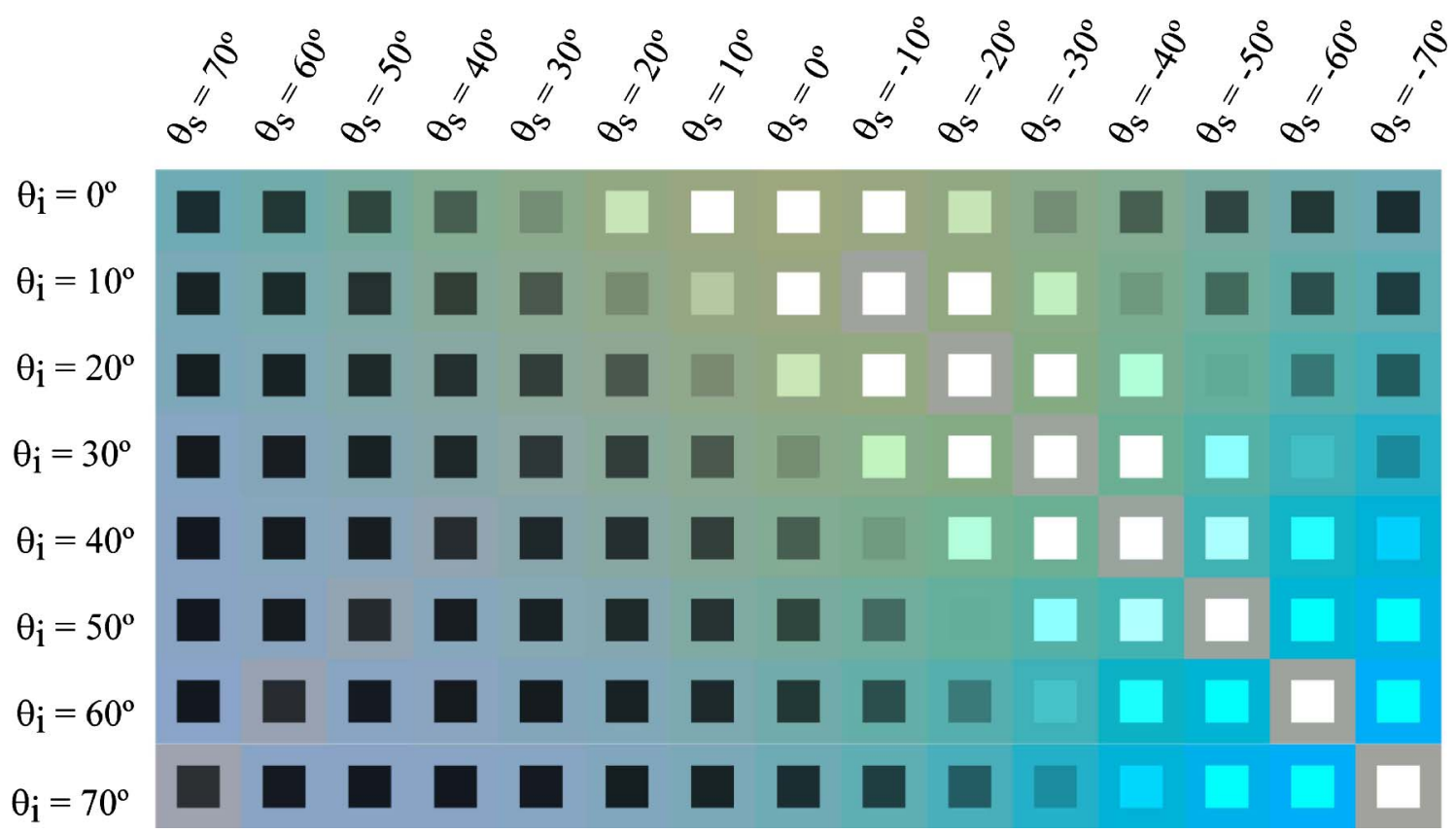

(a)

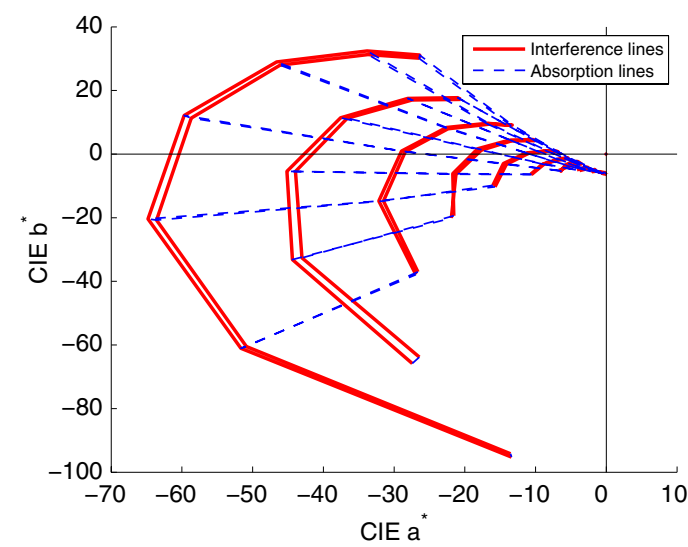

(b)

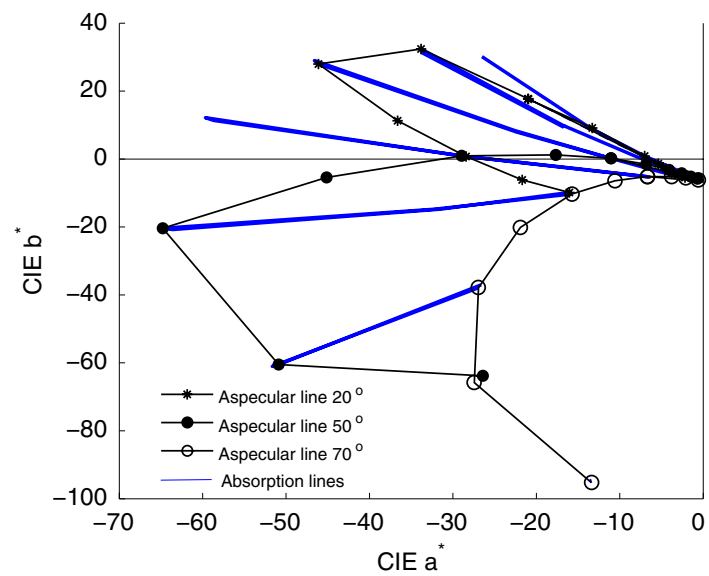

(c)

Fig. 4. (a) Representation of the color gamut, (b) the $a^{*}, b^{*}$-diagram, and (c) the $a^{*}, b^{*}$-diagram with three aspecular lines for lapis sunlight (MC1).

$$
\begin{gathered}
\theta_{\mathrm{inc}} \sim \theta_{\mathrm{bi}}, \\
\theta_{\mathrm{asp}}=\theta_{i}+\theta_{s}, \\
\theta_{\mathrm{bi}}=\theta_{i}-\theta_{s},
\end{gathered}
$$

$$
\begin{aligned}
& \theta_{i}=\frac{1}{2}\left(\theta_{\mathrm{asp}}+\theta_{\mathrm{bi}}\right), \\
& \theta_{s}=\frac{1}{2}\left(\theta_{\mathrm{asp}}-\theta_{\mathrm{bi}}\right) .
\end{aligned}
$$

For the sake of clarity, the three types of lines (interference, absorption, and aspecular) are described in Table $\underline{1}$. 


\section{MATERIALS AND METHOD}

Six special effect coatings were analyzed in this work. Three of them are commercialized by Merck and the other three are prototypes from BASF Coatings. Their descriptions and types of interference pigment are reported in Table 2 .

In order to realize the representation with real coatings, we measured the spectral bidirectional reflectance distribution function (BRDF) [27] to calculate the color of every coating. The measurement of the spectral BRDF was performed by using the GEFE goniospectrophotometer [28], which was developed at the Optics Institute of Consejo Superior de Investigaciones Científicas (IO-CSIC). It consists of a fixed, uniform, collimated light source and a robotarm that places the sample, making it possible to automatically and simultaneously change irradiation and viewing directions. For the data acquisition, a CS-2000 A Konica Minolta spectroradiometer was employed. This device operates within the visible range (380-780 nm), performing spectral measurements without contact with the sample at a $1 \mathrm{~nm}$ sampling interval and with a $4 \mathrm{~nm}$ bandwidth. The spectral BRDF for every coating was sampled within the incidence plane $\left(\phi_{s}-\phi_{i}=0^{\circ}\right.$ and $\left.180^{\circ}\right)$ at combinations of $\theta_{i}$ and $\theta_{s}$, which take values ranging from $0^{\circ}$ to $70^{\circ}$ at steps of $10^{\circ}$.

The color coordinates at every geometry were calculated from the spectral BRDF measurements using the CIE-D65 illuminant and the CIE-1964 standard observer [29]. The color representation is accomplished by converting the CIELAB coordinates to sRGB digital values for every geometry. White central squares in the cells correspond to saturation very

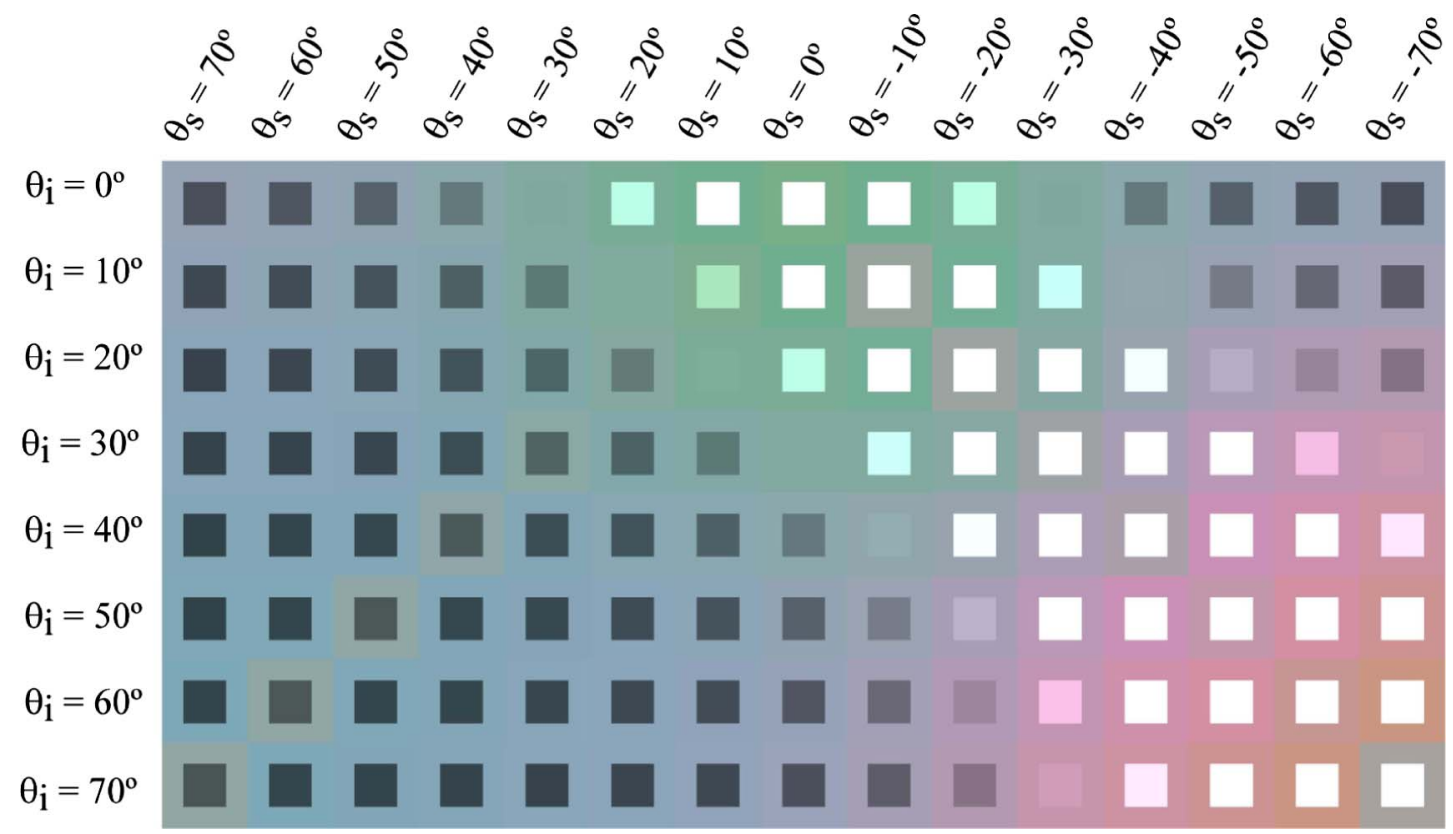

(a)

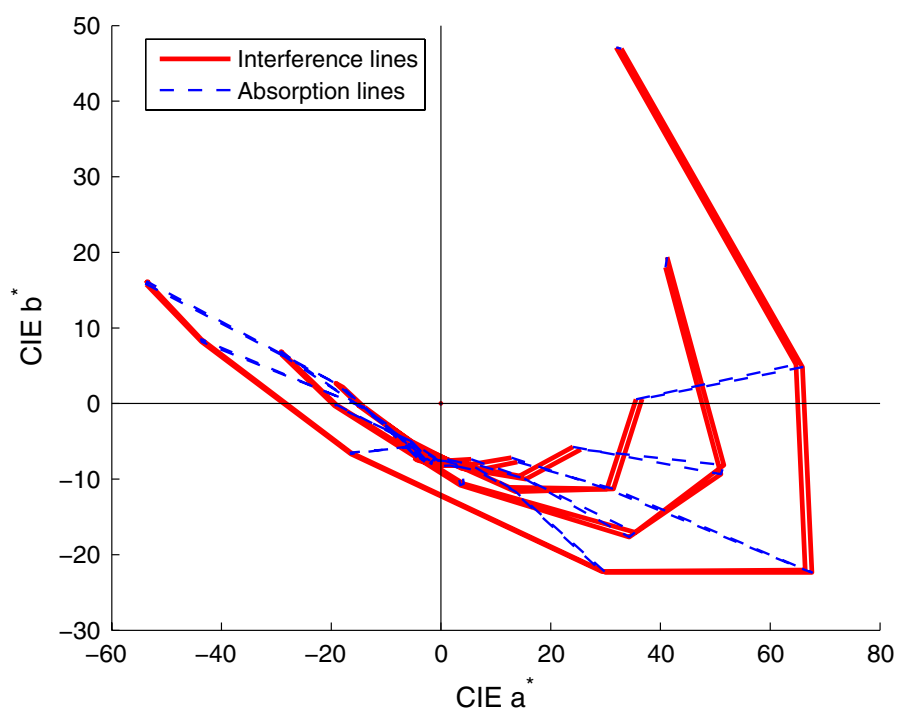

(b)

Fig. 5. (a) Representation of the color gamut and (b) the $a^{*}, b^{*}$-diagram for arctic fire (MC2). 
close to specular geometries, because digital sRGB values higher than 255 are obtained.

\section{RESULTS AND DISCUSSION}

The proposed representations of the color gamut of the studied coatings and the $a^{*}, b^{*}$-diagrams are shown in Figs. $\underline{4}-\underline{9}$, in parts (a) and (b), respectively. An additional $a^{*}, b^{*}$-diagram for Lapis Sunlight coating [Fig. 4(c)] is shown, where several absorption lines and three aspecular lines $\left(\theta_{i}=20^{\circ}, 50^{\circ}\right.$, and $70^{\circ}$ ) are plotted in order to compare their hue angle variations. It is clear that, while absorption lines have only a slight variation of the hue angle (mostly due to the existence of absorption pigments), the aspecular lines present a much stronger variation.
Regarding the representation of the color gamut, it should be noticed how clearly the hue variations on the diagonals of the corresponding interference lines of $\theta_{\text {asp }}=-10^{\circ}$ and $10^{\circ}$ are seen. The further the diagonals lie from this diagonal, the less the hue variation is along the length of them, toward the constant hue representing the absorption pigments. The maximum chroma variation is observed on the diagonals of the corresponding absorption lines, increasing toward the specular diagonal. The $\mathrm{a}^{*}, \mathrm{~b}^{*}$-diagrams allow the color parameters to be quantified. In addition, they show the convenience of using the interference and absorption lines (continuous red and discontinuous blue lines, respectively) to characterize the variation of the visual attributes of these coatings. Absorption lines present a strong variation of chroma, whereas interference lines present a strong variation of hue angle. Cis and

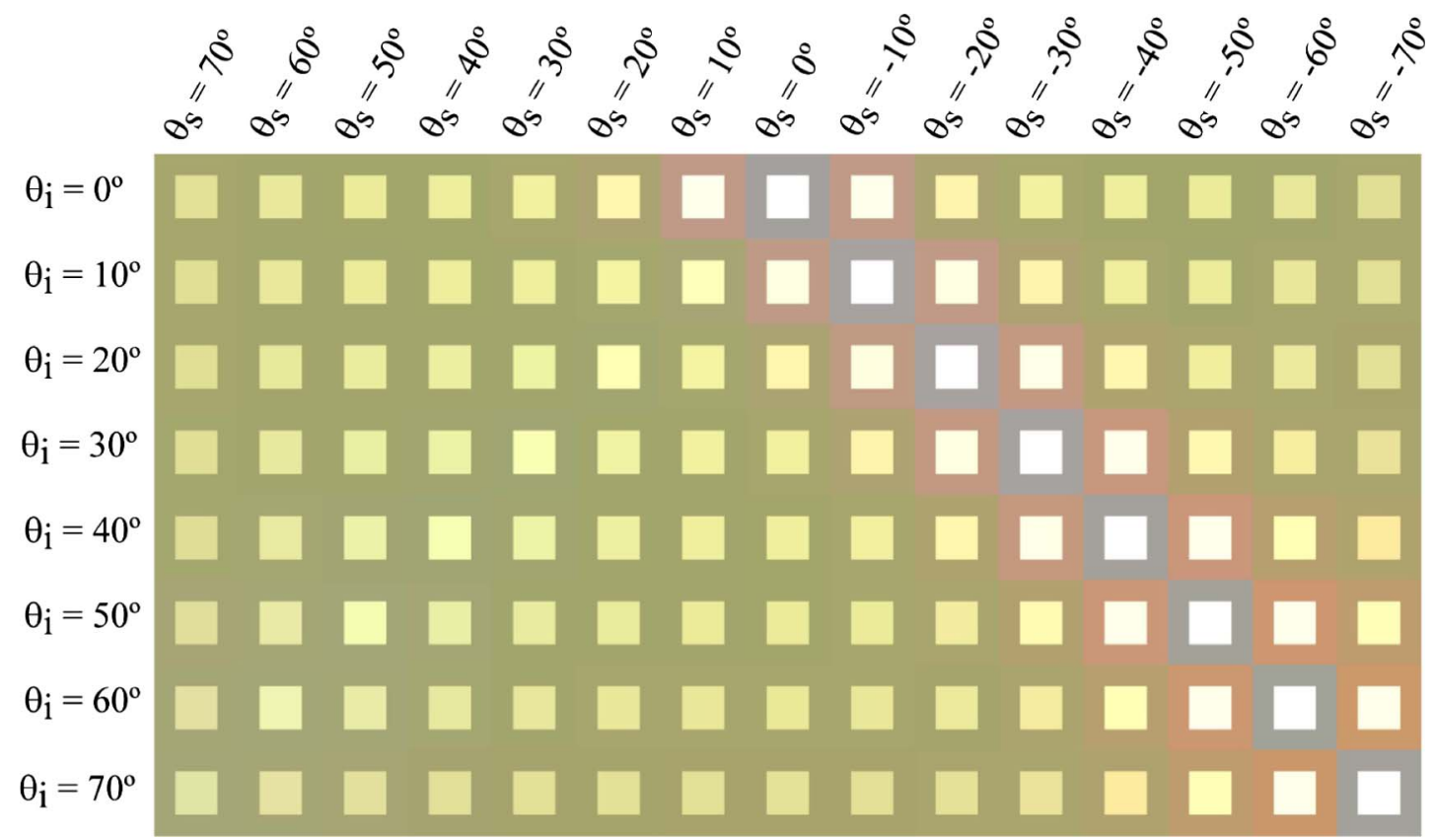

(a)

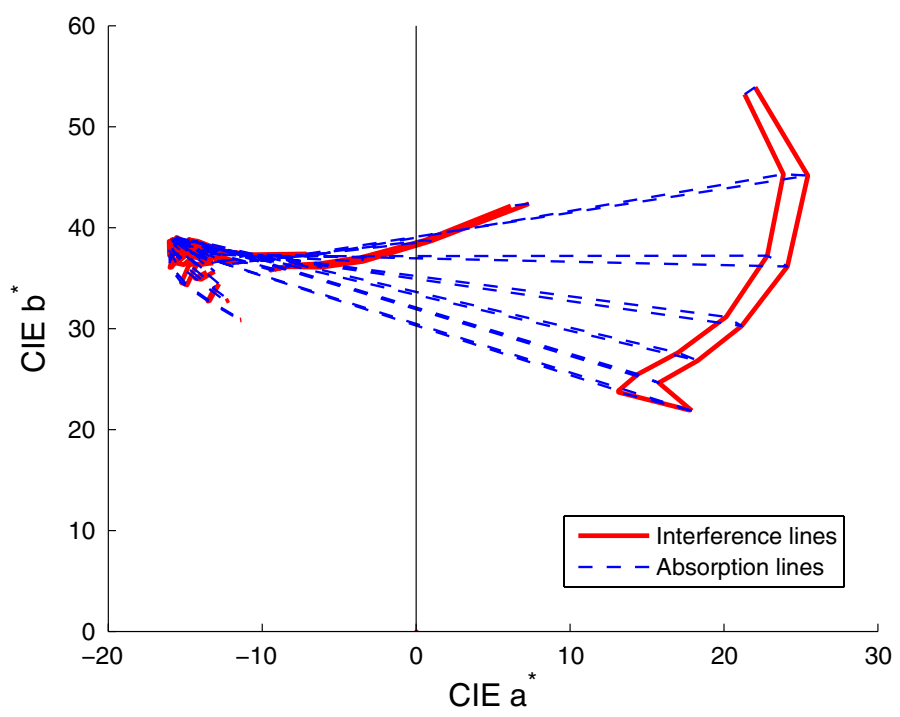

(b)

Fig. 6. (a) Representation of the color gamut and (b) the $a^{*}, b^{*}$-diagram for light yellow and solaris red (MXi). 
trans interference lines are almost identical, although a slight separation between them can be detected.

We have inferred from the flake-based approach to these coatings a general interpretation in terms of the mixture of one characteristic pattern of the absorption pigments and another characteristic pattern of the interference pigments in $\mathrm{a}^{*}$, $b^{*}$-diagrams. The prevalence of one of these patterns over the other in a certain area of the diagram means that, for those geometries, the corresponding mechanism of reflection prevails over the other. These patterns are clearly outlined from less to more complexity in Fig. 10.

The general color shift of absorption pigments in transparent layers is shown in Fig. 10(a). The black ellipses represent the range of variation of two coatings (A1 and A2). All points are distributed within a small area of almost constant hue. The chroma depends on the probability $P_{a}$ that an impinging photon reached an absorption pigment, and it depends on the density of pigments or on the thickness of the layer. In the case of Fig. 10(a), the coating A2 has a higher probability $P_{a}$ than coating A1.

The general color shift of metallic pigments in transparent layers is shown in Fig. 10(b), where the behavior of two coatings (M1 and M2) is outlined. Although this range is also distributed within an area of almost constant hue, the area is extended around a much longer line since there is a high variation of chroma, which is the main characteristic of these coatings. Its length allows several groups (big black circles) to be distinguished. Every group is characterized by a constant absolute value of the aspecular angle. The lower the aspecular angle, the more is the prevalence of the specularly reflected light at the flakes over the diffused light from the absorption pigments and the higher the flake-related chroma. In addition,

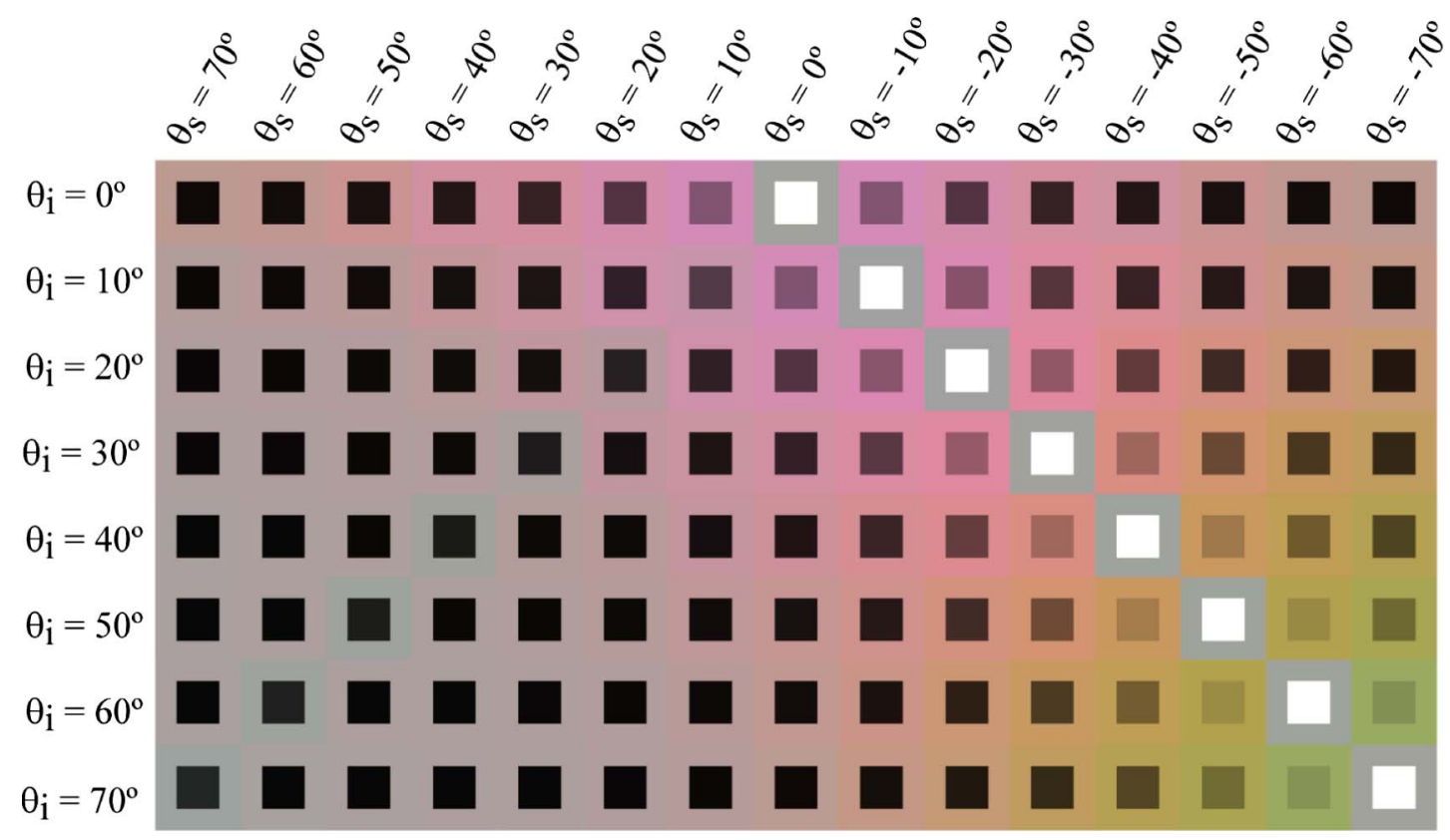

(a)

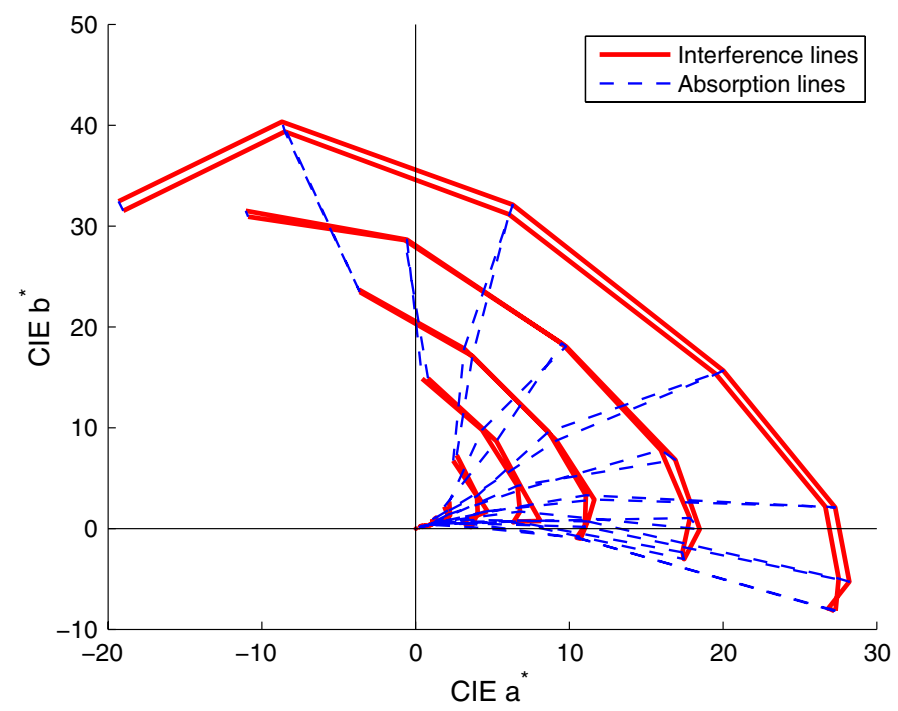

(b)

Fig. 7. (a) Representation of the color gamut and (b) the $a^{*}, b^{*}$-diagram for burgundy and olive green coating (BASF1). 
the narrower the orientation distribution of the flakes [ $\left.D\left(\theta_{\text {flake }}\right)\right]$, the more separated these groups are at low aspecular angles since more flakes are contributing to the reflection. The coating M1 would present a higher chroma and a glossier appearance than M2.

The general color shift of interference pigments in transparent layers is shown in Fig. 10(c), where the behavior of two coatings (S1 and $\mathrm{S} 2$ ) is outlined. Something similar to the previous case happens, but here the elements of every group are almost circularly distributed around the origin. These groups are marked in the plot by continuous lines which represent the interference lines. On the other hand, the discontinuous lines represent the absorption lines of constant hue. The coating S1 would present a glossier appearance than S2. However, the latter present a higher hue variation, as can be deduced by observing the hue angle variations at the most external interference lines.

When absorption and interference pigments are embedded together in a coating, their spectral reflectances are combined at the different geometries. It is not possible to predict the color shift in this case without knowing these spectral reflectances, but it is reasonable to think that reflective color due to the absorption dominates over color due to reflection from interference pigments at some geometries and that the opposite also happens, as outlined in Fig. 10(d), where a coating with a mixture of $\mathrm{A} 2$ and $\mathrm{S} 1$ is simulated. At intermediate geometries (no prevalence of any pigment-type), the interference and absorption lines are deformed. Then it can be said that the prevalence of both special effect reflection and scattered light by the absorption pigments is characterized by a

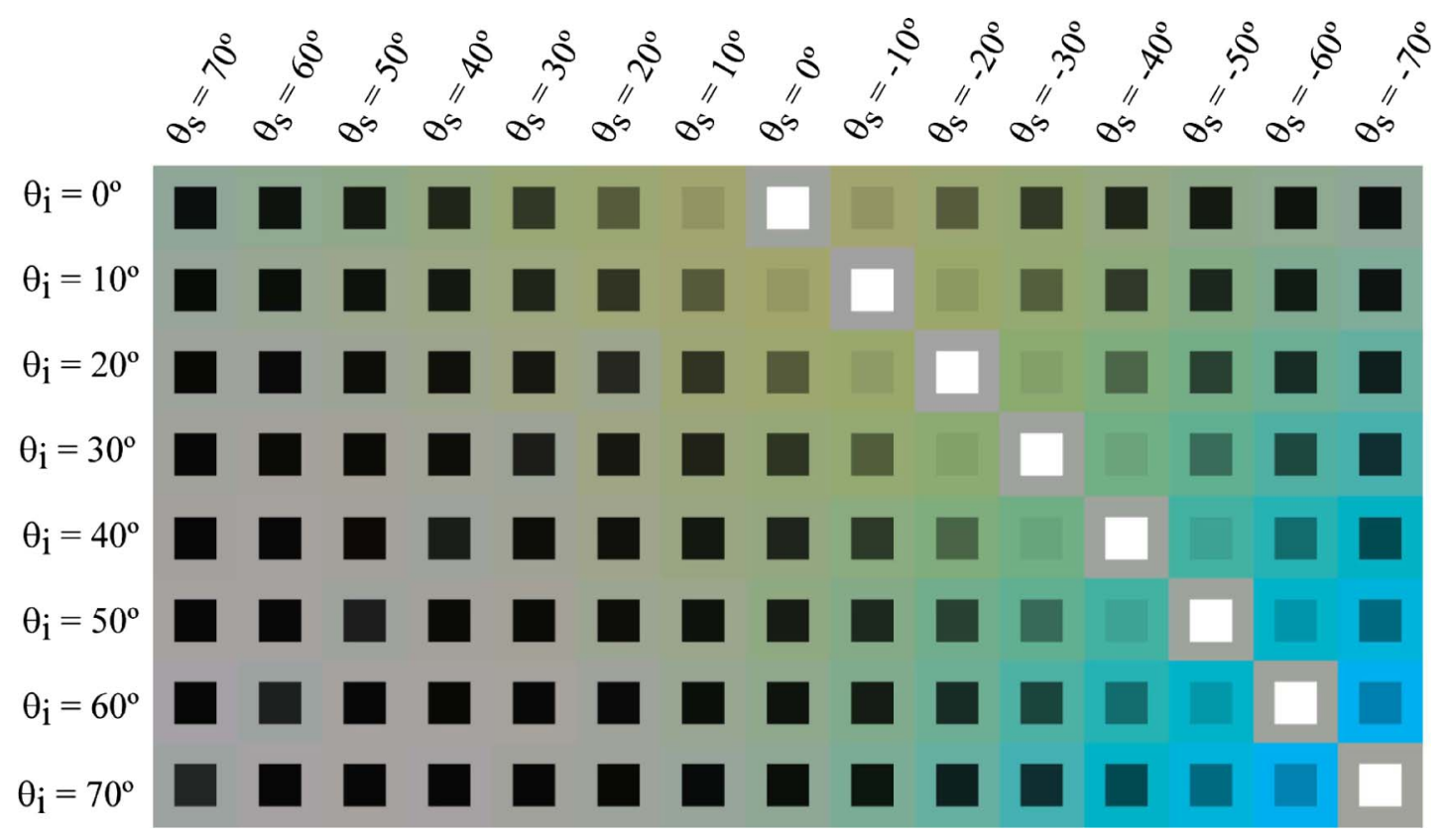

(a)

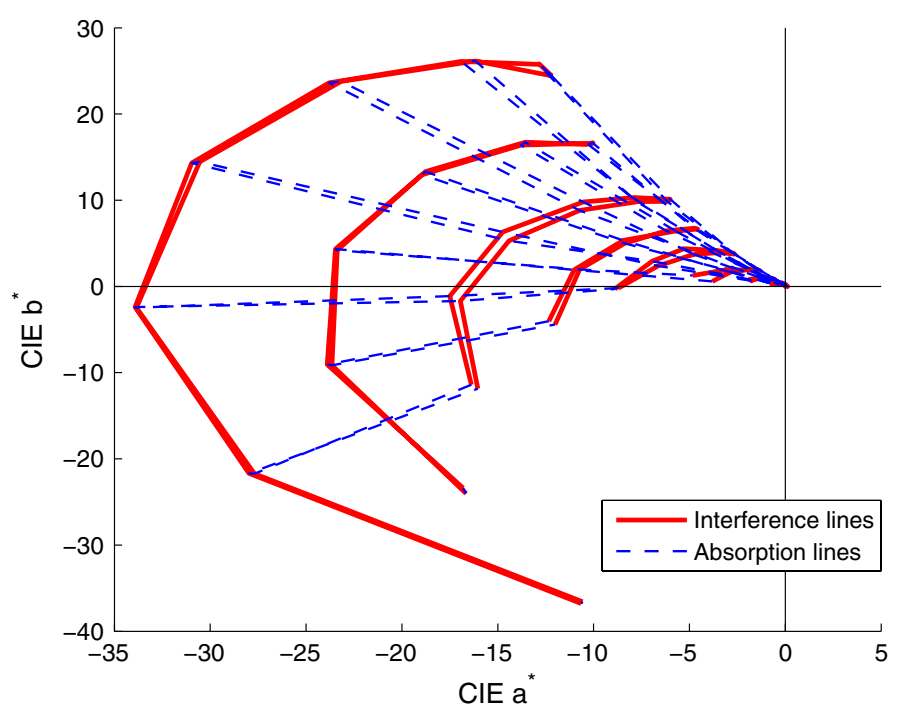

(b)

Fig. 8. (a) Representation of the color gamut and (b) the $a^{*}, b^{*}$-diagram for laurel green and greenish blue coating (BASF2). 
constant hue along the length of the absorption lines in special effect coatings, the difference being that the special effect reflection dominates at low aspecular angles and that the scattered light by the absorption pigments dominates at higher ones.

Real coatings in Figs. $\underline{4}-\underline{9}$ show the described behavior which can be synthesized and quantified by simple observation of interference and absorption lines.

It has been claimed in earlier publications $[\underline{17}, \underline{18}]$ that the interference lines with low aspecular angles characterize the interference pigment present in the coating. It was recently proved by the thorough study by Kirchner and Cramer [22] and it agrees with our discussion. It should be noted that it occurs at aspecular angles where the special effect reflection dominates the scattered light from by absorption pigments.
That is, when there are enough flakes with the optimal slope.

A characterization that can be seen from the interference lines with low aspecular angles is the hue angle variation. It is shown for all coatings in Table $\underline{3}$ as the accumulated hue angle variation with $\theta_{\text {asp }}=10^{\circ}$. For the MC1, MC2, BASF1, and BASF2 coatings, the hue angle variation along the length of the interference line at low aspecular angles is identical to the total hue angle variation, showing the prevalence of the color due to interference pigments over the color due to absorption pigments for any geometry. The case is different for MXi and BASF3 coatings, where the color due to absorption pigments prevails for geometries with high aspecular angles. In these two cases, the interference line at low aspecular angles does not account for the total hue angle

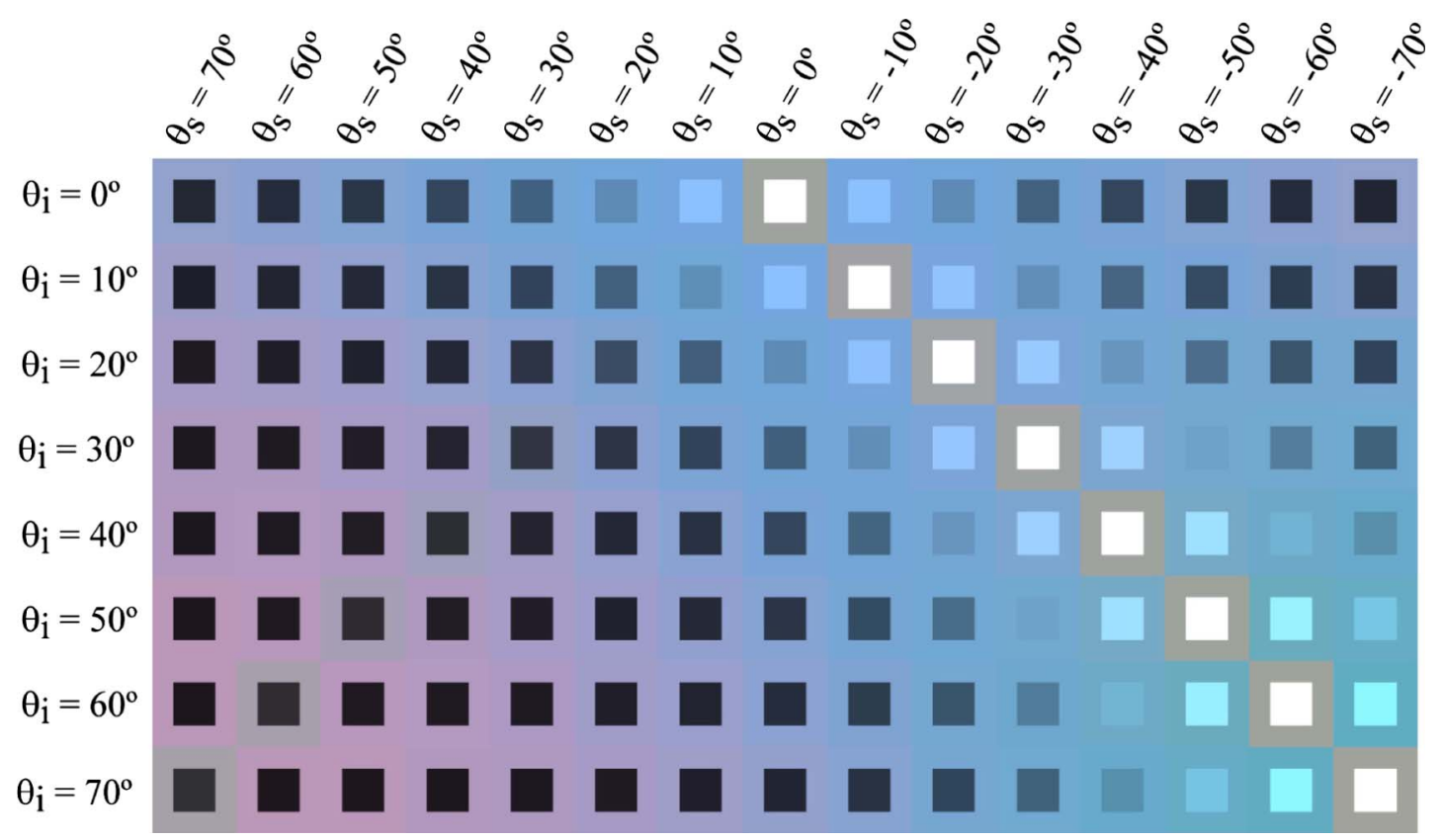

(a)

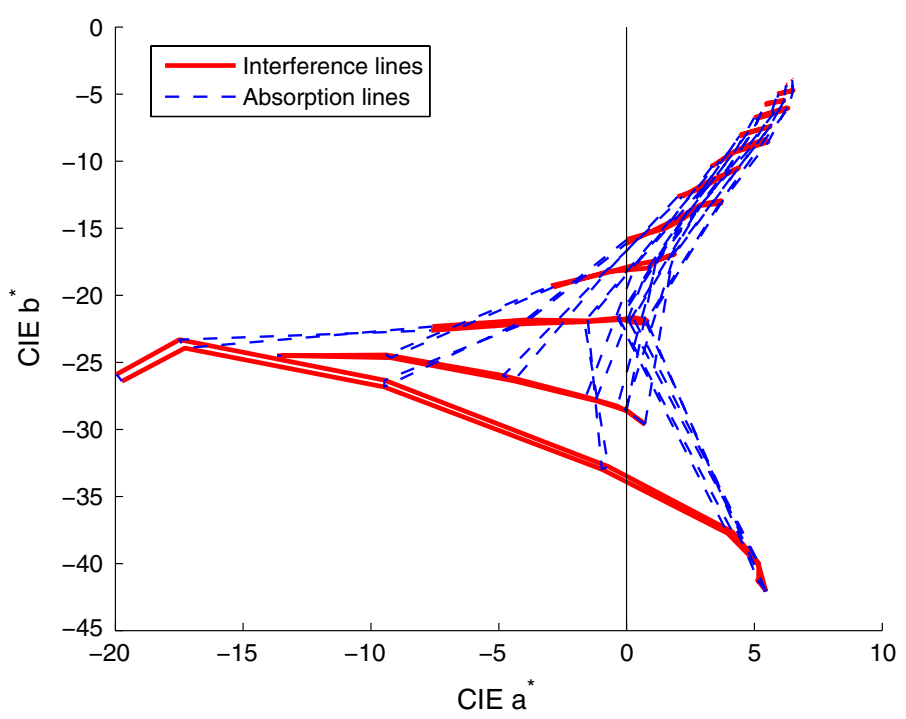

(b)

Fig. 9. (a) Representation of the color gamut and (b) the $a^{*}, b^{*}$-diagram for light blue and pale turquoise coating (BASF3). 


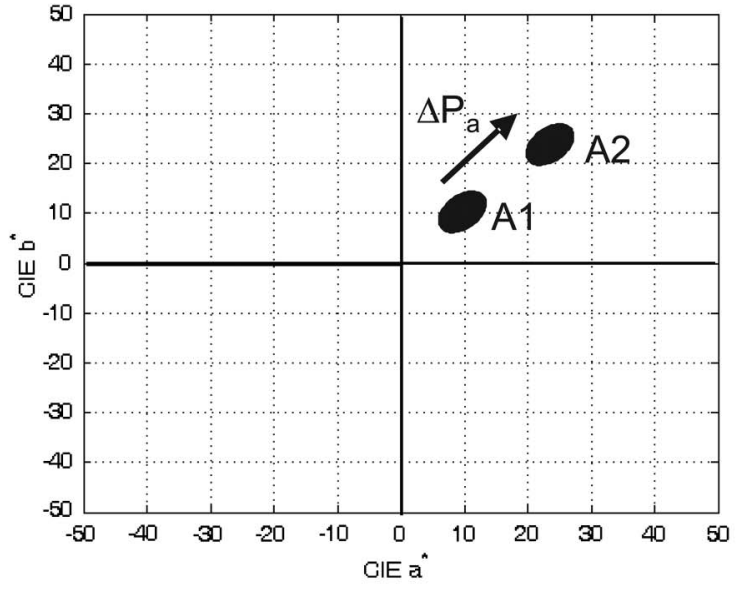

(a)

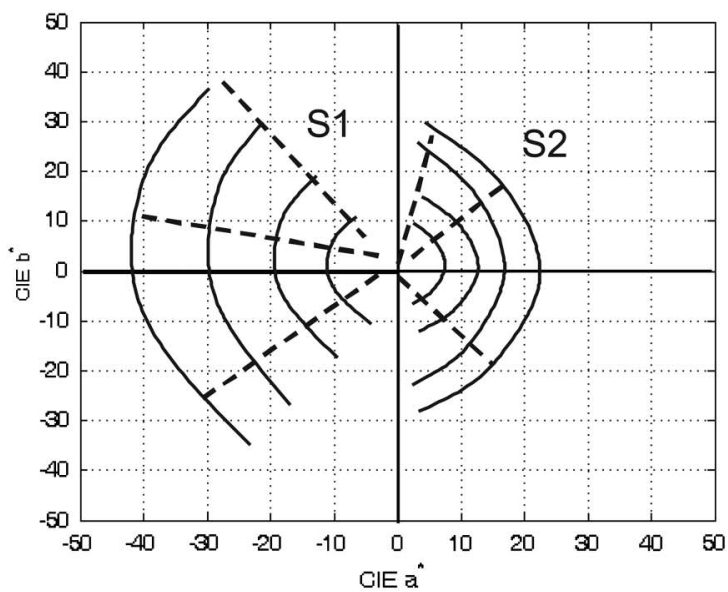

(c)

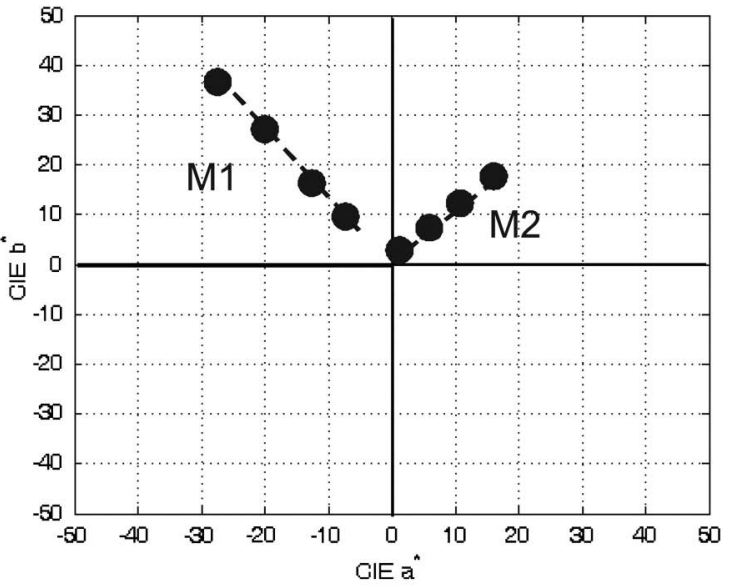

(b)

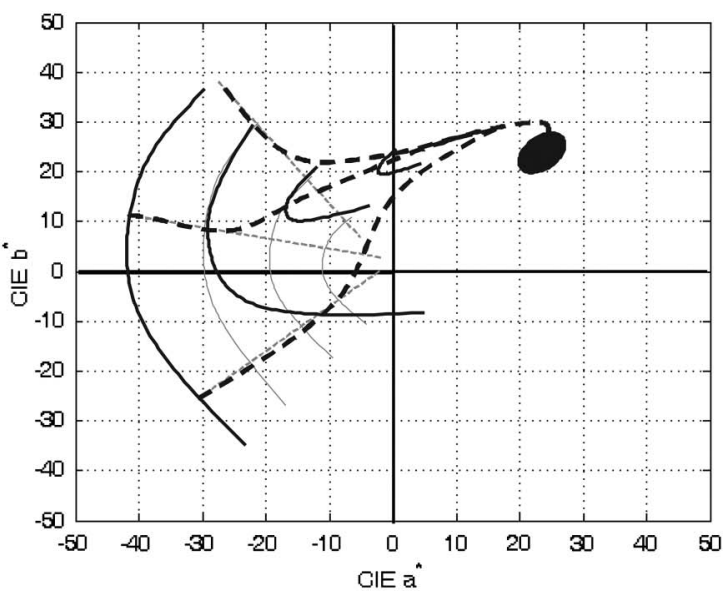

(d)

Fig. 10. Outline of the general color shift of effect coatings. (a) Absorption pigment color shift. (b) Metallic pigment color shift. (c) Special effect pigment color shift. (d) A possible color shift for a coating with a mixture of A2 and S1 pigments.

variation of the coating. Considering the shape of the interference (not circularly distributed around the origin) and the variable hue angle on the absorption lines in Fig. 6(b) (MXi), it is very difficult to affirm that any of the shown interference lines characterize the special effect coating. On the contrary, it seems that the contribution of the absorption reflection is always relevant in this coating. The opposite cases are those in Fig. 4(b) (MC1), 7(b) (BASF1), and 8(b) (BASF2). Although, it would be possible to affirm that MC1 and BASF2 contain the same or very similar interference pigment. The interference lines with the lowest aspecular angles are not completely circular or with constant chroma simply because the spectral variability of the spectral reflectance of the flake changes with the incidence angle on it.

The inspection of absorption lines provides information about special effect coatings in twofold: chroma variation due to the interference pigments and relative contribution of the absorption pigments to the color at a specific geometry. Chroma variations of the studied coatings along the length of the absorption line with $\theta_{\mathrm{bi}}=10^{\circ}$ are shown in Fig. 11(a) as a function of $\theta_{\text {asp }}$. This absorption line was chosen because it provides a high number of measurement geometries. Except for the MXi coating, all coatings present decreasing chroma variation from low aspecular angles to higher ones, from a very abrupt decrease to a tendency of constant value. Since the aspecular angles are related to $\theta_{\text {flake }}[$ Fig. $3(\mathrm{~b})]$ and the absorption lines represent the color variation at constant $\theta_{\text {inc, }}$, the curves in Fig. 11(a) can be almost directly related to the orientation distribution of the flakes. The chroma variation from the lowest aspecular angle to the highest one can be regarded as a rough descriptor of this distribution. In addition, the constant value to which the chroma tends, taken as the value at the highest $\theta_{\text {asp }}$, can be used to describe the amount of absorption pigments. These two descriptors are shown in Table 3 for all coatings (as "Chroma Variation" and "Absorption Chroma"). The curve of the MXi coating is quite different. The interpretation in the terms used here is that there are a high proportion of absorption pigments and, unfortunately, anything can be said about the orientation distribution of the Xirallic flakes.

The hue angle variation along the length of the absorption lines gives insight into the dominance of either the interference pigments or the absorption pigments [see Fig. 11(b) with $\theta_{\mathrm{bi}}=10^{\circ}$. If the hue angle is almost constant at low aspecular angles, special effect reflection prevails, and the contrary happens if the hue angle is almost constant at high aspecular angles. Thus, we think that the absorption lines can characterize the scattering by the absorption pigments, as it was 


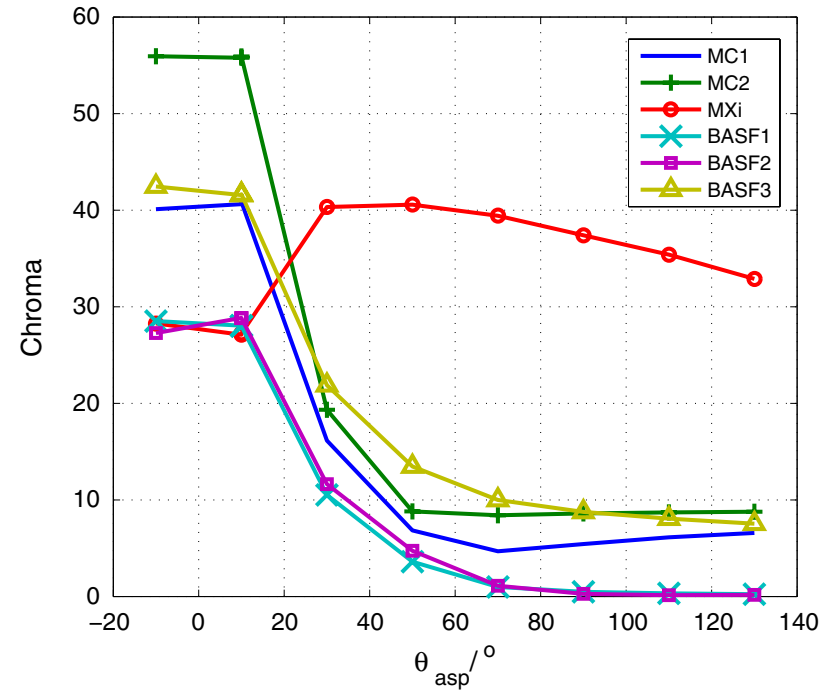

(a)

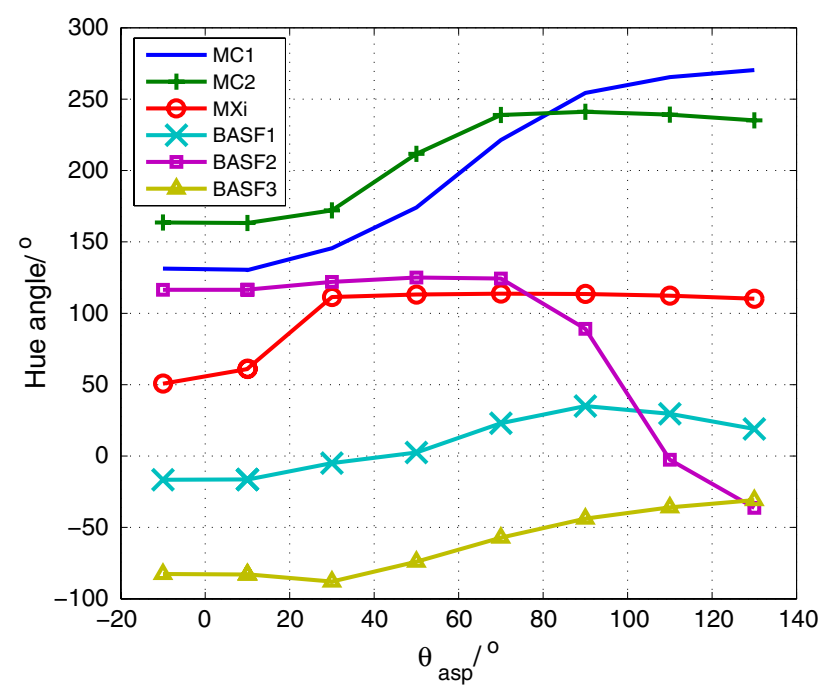

(b)

Fig. 11. (a) Chroma and (b) hue angle variation along the length of the absorption line with $\theta_{\mathrm{bi}}=10^{\circ}$ of studied coatings.

claimed for the aspecular lines in the past [17,22]. From lower to higher angles, the minimum $\theta_{\text {asp }}$ at which the hue angle is not constant anymore along the length of the absorption line can be used to describe how much overall contribution the absorption pigment reflection has. This descriptor, named $C_{A}$, is shown in the last column in Table 3.

Table 3. Values of the Proposed Descriptors to Characterize Special Effect Coatings

\begin{tabular}{lcccc}
\hline Coating & $\begin{array}{c}\text { Hue Angle } \\
\text { Variation }\left({ }^{\circ}\right)\end{array}$ & $\begin{array}{c}\text { Chroma } \\
\text { Variation }\end{array}$ & $\begin{array}{c}\text { Absorption } \\
\text { Chroma }\end{array}$ & $C_{\mathrm{A}}\left(^{\circ}\right)$ \\
\hline MCS1 & 131.7 & 36.2 & 6.6 & 10 \\
MCS2 & 251.8 & 47.5 & 8.8 & 10 \\
MXi & 17.2 & 13.5 & 32.9 & 10 \\
BASF1 & 137.5 & 28.3 & 0.24 & 10 \\
BASF2 & 137.4 & 28.7 & 0.17 & 70 \\
BASF3 & 46.0 & 34.9 & 7.5 & 30 \\
\hline
\end{tabular}

The Xirallic coating (MXi) is the most different among the studied coatings. The special effect can be clearly observed only at low aspecular angles, but the absorption pigment reflection highly contributes at all geometries, providing the yellow hue [see Fig. 6(a)].

\section{CONCLUSIONS}

A new representation of the color gamut for special effect coatings is proposed. The most important characteristic of the proposed representation is that it allows for a straightforward understanding of the color shift in these coatings to be done both in terms of conventional irradiation and viewing angles and in terms of flake-based parameters. This representation allows the color shift of special effect coatings to be figured out which is a very interesting alternative to the usage of goniovision devices to perceive the color gamut.

We have previously proven that the bistatic angle is almost proportional to the incidence angle on the flakes. Therefore, a different line was proposed to assess the color shift of special effect coating on $a^{*}, b^{*}$-diagrams: the absorption line. Similar to interference and aspecular lines (constant aspecular and irradiation angles, respectively), an absorption line is the locus of calculated color coordinates from measurement geometries with a fixed bistatic angle. It gives insight into the relative contributions of the absorption and interference pigments at different geometries in a more direct way than the aspecular lines.

The proposed representation of the color gamut was realized for six different special effect coatings: three from Merck and three from BASF Coatings. These representations visually show the color for a meaningful number of geometries and could be used, either in an extended or a simplified way, as a basis for color cataloging these kinds of coatings, visualized in colorimetrically calibrated displays or printing devices. On the other hand, $a^{*}, b^{*}$-diagrams allow a more quantitative analysis to be done. The representations of hue angle and chroma along the length of the proposed absorption lines show the convenience of using these lines to understand the relative contributions of the scattering at the absorption and interference pigments to the spectral BRDF at different geometries. Descriptors were proposed in these terms which may help in the quality control and design of new color recipes for these special effect coatings.

It should be noted that although absorption and interference lines allow the variation of perceptual attributes to be very clearly assessed, $\theta_{\text {inc }}$ or $\theta_{\text {flake }}$ are almost constant along the length of these lines and therefore measurements restricted only to one of them would provide some spectral or angular redundant information. For this reason, when the question is to select a reduced number of nonredundant measurement geometries to completely characterize special effect coatings, to lie only on these lines is not adequate. For instance, in a previous work [23] we proposed a set of nine measurement geometries organized in two aspecular lines with $\theta_{i}=20^{\circ}$ and $50^{\circ}$ which provide nonredundant information about variations of chroma, hue, and lightness and allow spectral reflectance at any other geometries to be estimated using a PCA-based procedure. This kind of organization in aspecular lines is also common in commercial portable instruments. 


\section{ACKNOWLEDGMENTS}

The authors are grateful to the "Plan Nacional de Física" for funding this work FIS2010-19756-E, to CSIC's JAE Program, and the "European Social Fund" for awarding us a research grant. This study was also supported by the Spanish Ministry of Economy and Competitiveness under Grant No. DPI201130090-C02-02 and the European Union. The authors have no interest in the development or marketing of any product mentioned in this study.

\section{REFERENCES}

1. G. Pfaff and P. Reynders, "Angle-dependent optical effects deriving from submicron structures of films and pigments," Chem. Rev. 99, 1963-1982 (1999).

2. F. J. Maile, G. Pfaff, and P. Reynders, "Effect pigments-past, present and future" Prog. Org. Coat. 54, 150-163 (2005).

3. H. J. Streitberger and K. F. Dössel, Automotive Paints and Coatings (Wiley-VCH, 2008).

4. M. E. Nadal and E. A. Early, "Color measurements for pearlescent coatings," Color Res. Appl. 29, 38-42 (2004).

5. G. Baba, "Gonio-spectrophotometric analysis of pearl-mica paint," Die Farbe 37, 99-110 (1990).

6. T. A. Germer and M. E. Nadal, "Modeling the appearance of special effect pigment coatings," Proc. SPIE 4447, 77-86 (2001).

7. M. E. Nadal and T. A. Germer, "Colorimetric characterization of pearlescent coatings," Proc. SPIE 4421, 757-760 (2002).

8. B. Parker, "Color shift of light interference pigments," Surf. Coat. Aust. 39, 10-13 (2002).

9. F. Leloup, P. Hanselaer, M. Pointer, and J. Versluys, "Characterization of gonio-apparent colours," in Proceedings of AIC Color (AIC, 2005), pp. 515-518.

10. C. A. Nicholls, "Visual and instrumental characterisation of special-effect colours," Ph.D. thesis (University of Leeds, 2000).

11. R. Besold, "Metallic effect-characterization, parameter and methods for instrumentally determination," Die Farbe 37, 7985 (1990).

12. DIN 6175-2, "Tolerances for automotive paints, part 2: goniochromatic paints (Farbtoleranzen für automobillackierungenteil 2: effektlackierungen)," 2001.

13. E. Chorro, E. Perales, F. M. Martínez-Verdú, J. Campos, and A Pons, "Colorimetric and spectral evaluation of the optical anisotropy of metallic and pearlescent samples," J. Mod. Opt. 56, 1457-1465 (2009).

14. E. Kirchner, G. J. Van den Kieboom, L. Njo, R. Supér, and R. Gottenbos, "Observation of visual texture of metallic and pearlescent materials," Color Res. Appl. 32, 256-266 (2007).

15. W. R. Cramer, "Reflections on the right angle," Eur. Coat. J. 4, 32-37 (2012).

16. W. R. Cramer, "Examples of interference and the color pigment mixtures green with red and red with green," Color Res. Appl. 27, 276-281 (2002).

17. W. R. Cramer, "Der richtige blickwinkel: visuelle und instrumentelle abmusterung von interferenzpigmenten im vergleich," Farbe Lack 112, 26-30 (2006).

18. W. R. Cramer and P. W. Gabel, "Das gewisse etwas: dreiecksbeziehungen aus bunt-, aluminium- und interferenzpigmenten," Farbe Lack 109, 78-84 (2003).

19. W. R. Cramer, "Effekte sichten und beziffern: cis- und transgeometrien bei farbmetrischer beurteilung von effektpigmenten," Farbe Lack 108, 48-55 (2002)

20. W. R. Cramer and P. W. Gabel, "Effektvolles messen," Farbe Lack 107, 42-48 (2001).

21. W. R. Cramer and P. W. Gabel, "Measuring special effects," Paint. Coat. Ind. 9, 36-46 (2001).

22. E. Kirchner and W. Cramer, "Making sense of measurement geometries for multi-angle spectrophotometers," Color Res. Appl. 37, 186-198 (2012).

23. A. Ferrero, A. Rabal, J. Campos, F. Martínez-Verdú, E. Chorro, E. Perales, A. Pons, and M. L. Hernanz, "Spectral BRDF-based determination of proper measurement geometries to characterize color shift of special effect coatings," J. Opt. Soc. Am. A, 30, 206-214 (2013).

24. A. Ferrero, J. Campos, A. M. Rabal, A. Pons, M. L. Hernanz, and A. Corróns, "Principal components analysis on the spectral bidirectional reflectance distribution function of ceramic colour standards," Opt. Express 19, 19199-19211 (2011).

25. A. Ferrero, J. Campos, A. M. Rabal, A. Pons, M. L. Hernanz, and A. Corróns, "Variables separation of the spectral BRDF for better understanding color variation in special effect pigment coatings," J. Opt. Soc. Am. A 29, 842-847 (2012).

26. ASTM E 2539-08, "Standard practice for multiangle color measurement of interference pigments" (American Society for the Testing of Materials, 2009).

27. F. E. Nicodemus, J. C. Richmond, and J. J. Hsia, "Geometrical considerations and nomenclature for reflectance," Natl. Bur. Stand. (U.S.), Monogr. 160, 1-7 (1977).

28. A. M. Rabal, A. Ferrero, J. Campos, J. L. Fontecha, A. Pons, A. M Rubiño, and A. Corróns, "Automatic gonio-spectrophotometer for the absolute measurement of the spectral BRDF at in and out-of-plane and retroreflection geometries," Metrologia 49, 213-223 (2012).

29. P. Green and L. W. MacDonald, Colour Engineering: Achieving Device Independent Colour (Wiley, 2002). 